\title{
Cost-Effective Traction for Arthroscopic Shoulder Surgery
}

\author{
Ravi Kumar Ray', Helen Aung1, Steven Andrew Corbett ${ }^{2}$ \\ ${ }^{1}$ Orthopaedic Department, University Hospital Southampton NHS Foundation Trust, \\ Southampton, UK \\ ${ }^{2}$ Orthopaedic Department, Guy's \& St Thomas' Hospitals NHS Foundation Trust, \\ London, UK \\ Email: raviray@hotmail.com
}

Received 12 April 2014; revised 15 May 2014; accepted 23 May 2014

Copyright (C) 2014 by authors and Scientific Research Publishing Inc.

This work is licensed under the Creative Commons Attribution International License (CC BY). http://creativecommons.org/licenses/by/4.0/

(c) (i) Open Access

\section{Abstract}

The application of traction has enhanced views during arthroscopic shoulder surgery, easing visualization and accurate intervention within the shoulder joint and the subacromial space. Many innovative traction techniques are currently employed, including the use of padded traction equipment attached to boom arms and further, pedal-activated, hydraulic traction equipment. Variations in patient positioning and cost-benefit analysis of the use of traction in both beach chair and lateral decubitus positions have been performed in the literature. We demonstrate the use of readily available, simple and inexpensive resources in the beach chair position, allowing the application of traction with minimal set-up time and complexity. Initial equipment outlay requires a drip stand attached to a clamp at the end of the operating trolley, permitting a hook to be applied to the arm that is attached to traction cord and weights. We minimized the cost of ongoing consumable items per case to include a stockinette and benzoic tincture. The theatre team experiences a short learning curve associated with the ease of reliability and reproducibility of this technique.

\section{Keywords}

Cost, Traction, Shoulder, Arthroscopy, Beach-Chair

\section{Introduction}

The use of skin traction during arthroscopic shoulder surgery helps the joint to become distended, allowing better views intra-operatively. Access to the antero-inferior capsulo-labral complex during stabilizations may be 
compromised due to the surrounding soft tissues absorbing irrigation fluid. If the traction is maintained continuously, the surgeon is more likely to be able to complete the operation more speedily and without compromise. A similar situation arises when repairing rotator cuff tears in that the surgical field is continuously narrowing through soft tissue swelling. Traction allows a greater initial field of view thus permitting longer unimpeded surgical access.

It is well-recognized that traction is essential when placing patients in the lateral decubitus position for which boom arms are the preferred method. (Arthrex Snyder STaR SLEEVE, 3-point Shoulder Distraction System AR-1600M [1]). This relatively expensive equipment is attached to the operating trolley via clamps on the opposite side of the surgeon, putting the arm in a flexed abducted position thought to be optimal to gain good views whilst laying in a lateral position [2].

In a previous paper, comparing the lateral decubitus position to the beach chair position for shoulder arthroscopies, neither was shown to be superior with regards to setup, surgical visualization, access and risk [2]. However, we have selected the beach chair position as it negates the necessity to de-scrub, re-scrub and re-drape on switching to open surgery.

When the patient is in the beach chair position, although a mechanical arm holder can aid in supporting the arm, an assistant would otherwise be required to apply traction throughout the procedure [2].

Skin traction can be applied directly, using adhesive or non-adhesive padded strips applied on the dorsal and palmar aspects of the forearm, over which a bandage is tightened in a "herringbone" fashion to spread the load and avoid degloving. This bespoke traction set [3] contains the traction cord to which weights can then be applied over a pulley system.

Newer limb positioning equipment has become available [4] which suggests it can deliver unimpeded access to the shoulder through a hydraulic system that can be attached to the operating table. The forearm and hand are wrapped in well-padded single-use supports, which are subsequently attached to reusable metal rods themselves attached to the hydraulic equipment. When a pedal is depressed, the system becomes loose, allowing the surgeon to position the shoulder optimally. On release of the pedal, the hydraulic system engages, maintaining this position, whilst permitting one-handed maneuverability from the surgeon's perspective. The downside of this system lies in the initial expense and ongoing consumable costs.

In our study, we present a highly cost-effective method of applying skin traction during beach chair shoulder arthroscopy that minimizes consumables and setup time, whilst also negating the need for expensive hardware as an initial outlay.

\section{Materials \& Methods}

The equipment required to apply traction using our technique includes a clamp and a drip stand. The clamp is attached to the sidebar of the surgical trolley towards the foot-end of the bed and the drip stand is applied within the clamp with the top end of the drip stand being roughly level to the patient's head. Weights appropriate to the size of the patient are then attached to some traction cord and a steel hook. All metal equipment is reused once wiped clean. Roughly 2 feet $(60 \mathrm{~cm})$ of traction cord are required per patient, and subsequently disposed of.

The sterile consumables required include: 10 - $15 \mathrm{ml}$ of benzoic tincture (Friar's Balsam), and a stockinette, sized accordingly to the patient's arm.

The initial outlay includes the drip stand, clamp, hook, traction cord and adjustable weights.

Once our patients have been appropriately anaesthetized, they are transferred onto an operating trolley. In the beach chair position, we apply 10 degrees of Trendelenburg (head down) with 60 degrees of hip flexion and 20 degrees of knee flexion. We ensure adequate prevention of any undue pressure or traction injuries specifically by providing gel heel cups and by flexing the knees over a pillow to protect the sciatic and common peroneal nerves. Thromboembolic deterrent manoeuvers are also performed to include intermittent calf compression.

Whilst the patient is being sat up, the anaesthetist is ensuring safe and satisfactory head positioning, culminating in padded strapping of the head to the table.

The hand is prepared with an appropriate antiseptic agent then the surgeon then takes over holding the limb by applying a sterile stockinette over the hand. The remainder of the forearm, arm and shoulder are then prepared with the same agent. The forearm and arm is then prepared with one broad strip of benzoic tincture (Friar's Balsam) on each of the dorsal and ventral surfaces, taking care not to apply this circumferentially. Within a few seconds, this is sufficiently dry to roll the stockinette all the way up the arm, which is instantly adherent (see Figure 1 \& Figure 2). 


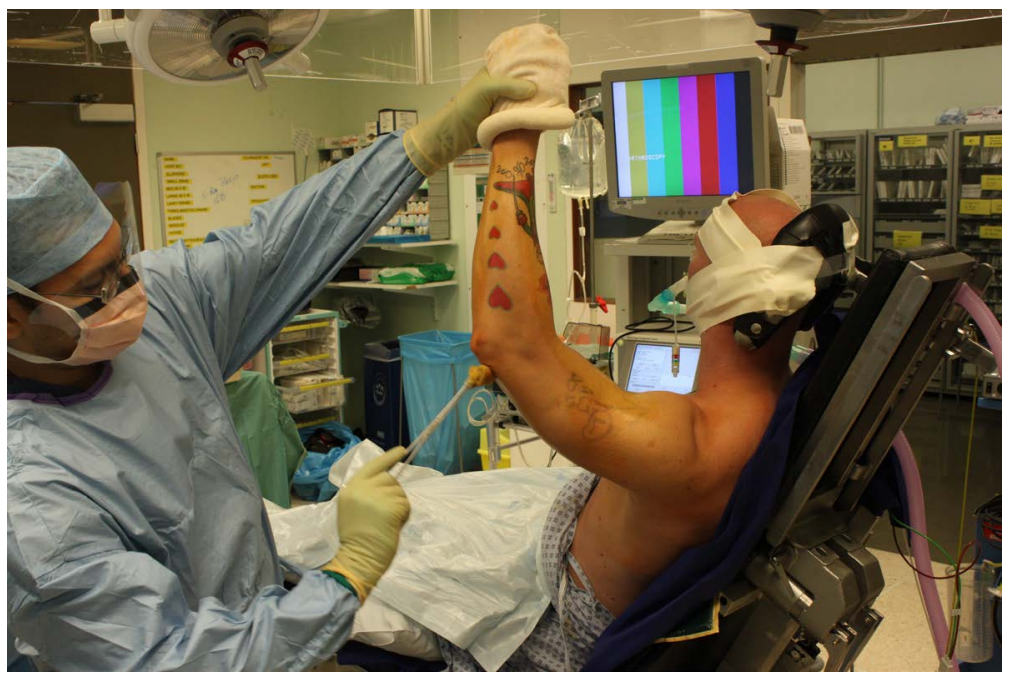

Figure 1. Preparation of the limb with Friar’s Balsam after skin preparation.

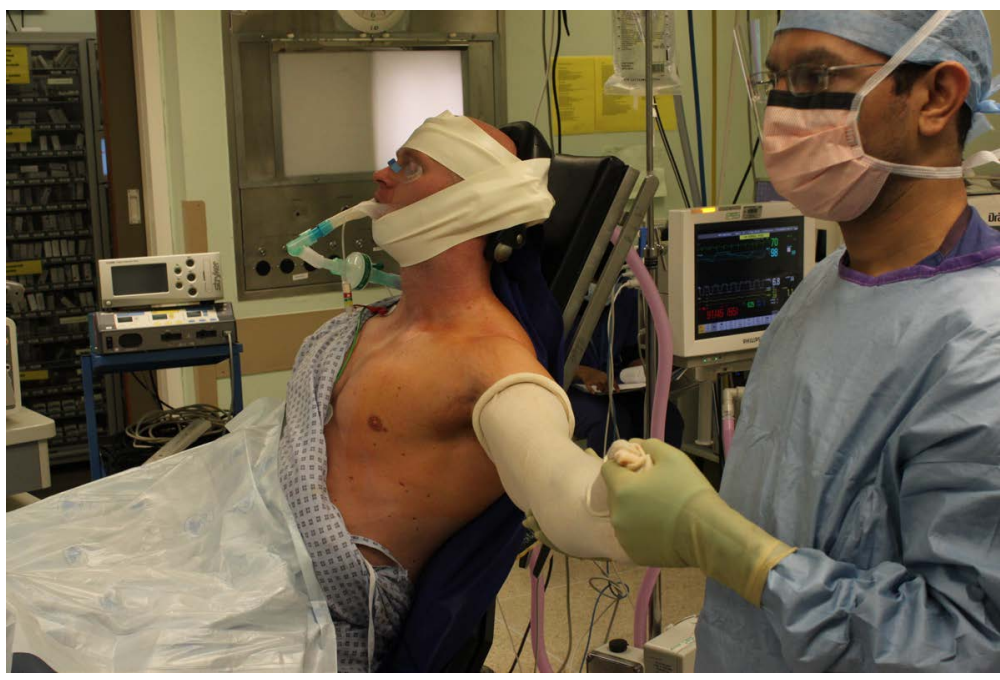

Figure 2. Stockinette applied over Friar's Balsam which is instantly adherent.

A small hole is made bluntly between the thumb and index finger through the stockinette, through which a non-sterile hook is applied. This is then attached to traction cord and weights over the pigtail-shaped hook of a drip stand, which acts as a pulley (see Figures 3-6).

In our institution, shoulder-specific drapes are used. These have adhesive strips towards the patient over the front, top and back of the shoulder with a "catch-bag" on the exterior. There is thus a necessity to dry the chest, neck and back to permit the attachment of these adhesive strips on the drapes. We combine the use of Friar's Balsam to these areas to enhance adhesion (see Figure 7).

A second, bar drape is then used to seal the arm, excluding the non-sterile hook and traction cord. Subsequent arthroscopic equipment can now be laid over the arm without concern for loss of sterility, whilst the surgeon is preparing arthroscopic surface markings and portal placement.

At the end of the procedure, the stockinette can be rolled easily down without concern for degloving.

\section{Results}

We have used this technique for a decade at Guy's and St Thomas' Hospitals without any allergic reactions to the benzoic tincture and no complications despite specifically looking for erythematous reactions, skin degloving, loss of traction or patient complaints regarding the odour. 


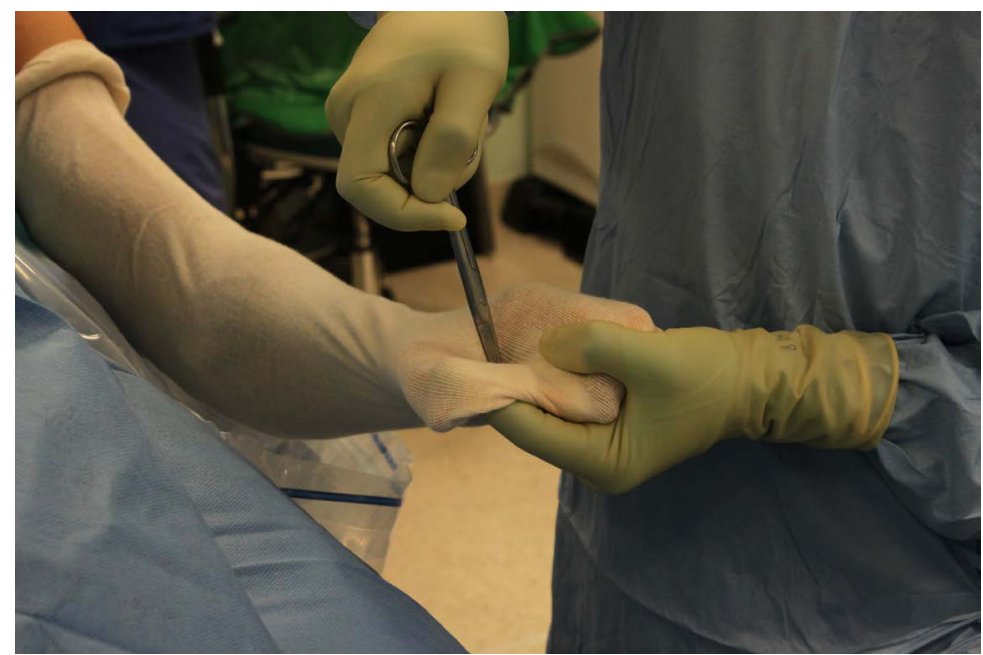

Figure 3. A hole is made bluntly through the space between thumb and index finger.

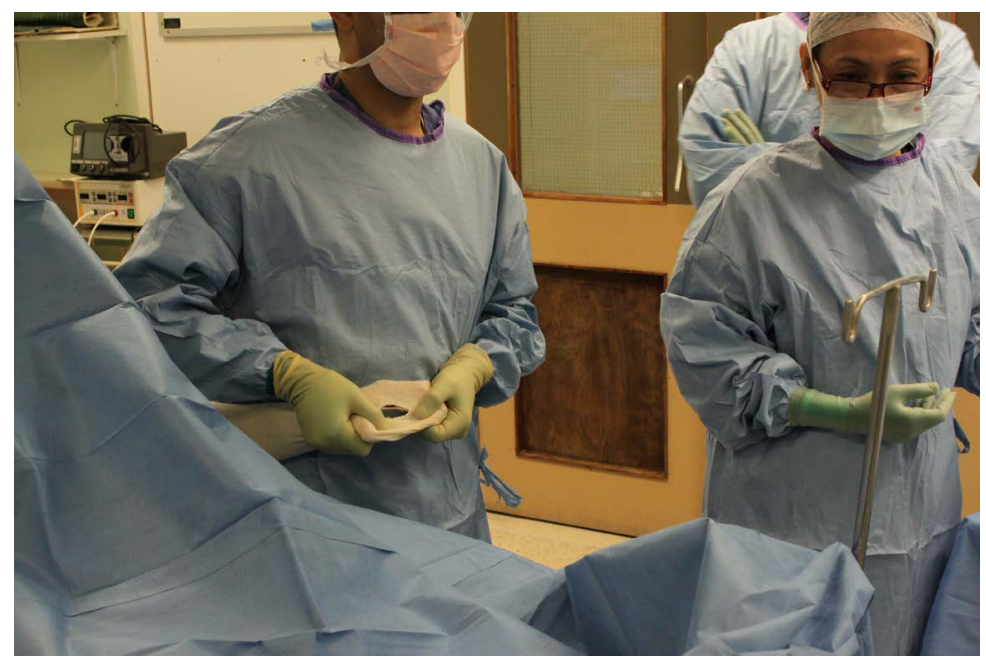

Figure 4. The hole is presented to unscrubbed theatre staff.

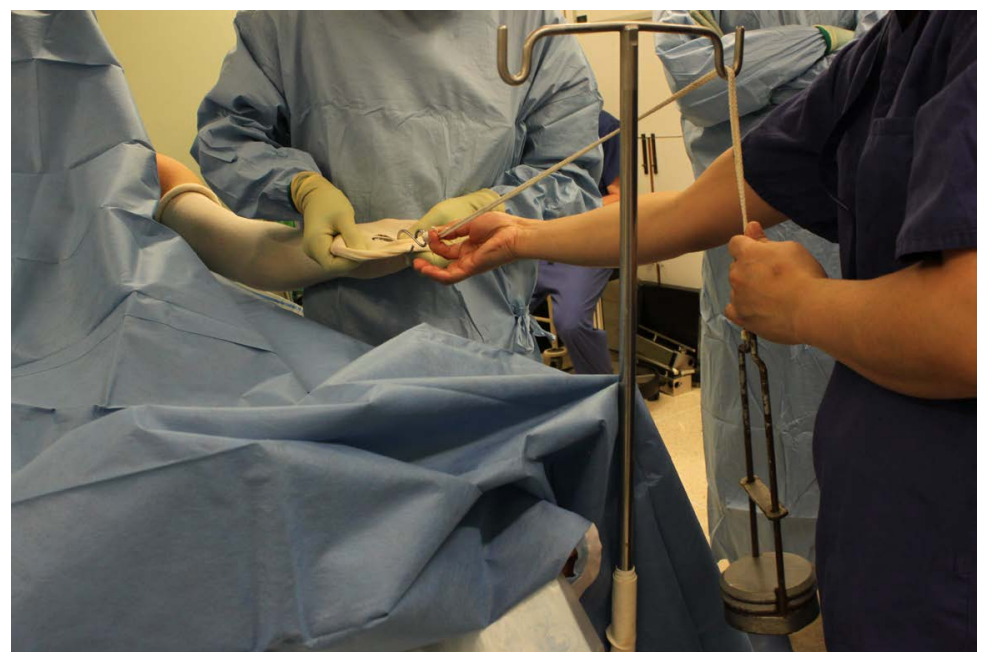

Figure 5. A hook is attached through the hole with weights for traction. 


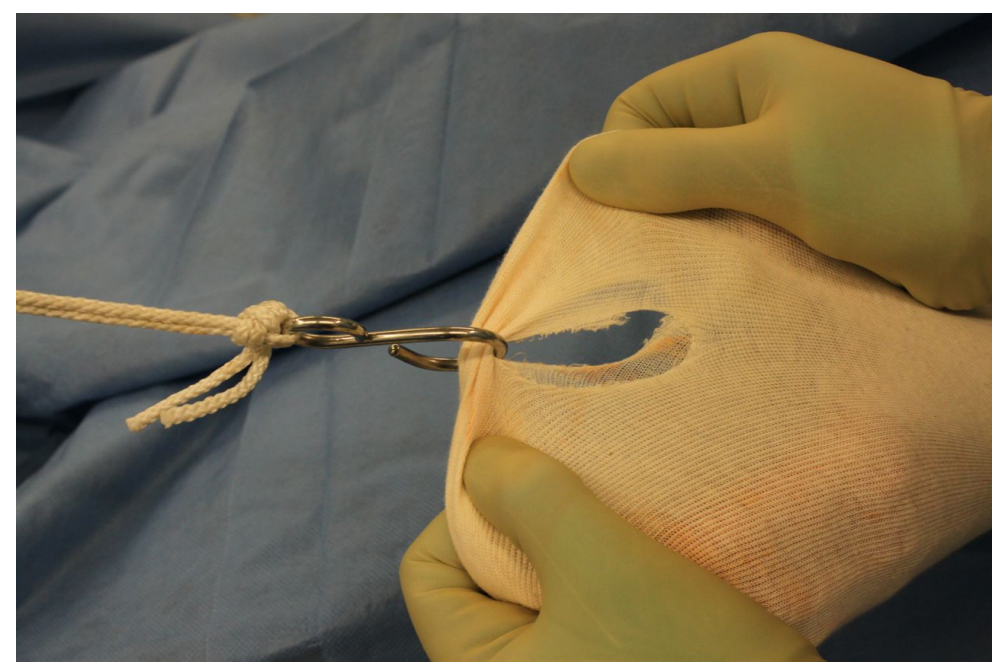

Figure 6. A hook is attached through the hole with weights for traction (close-up).

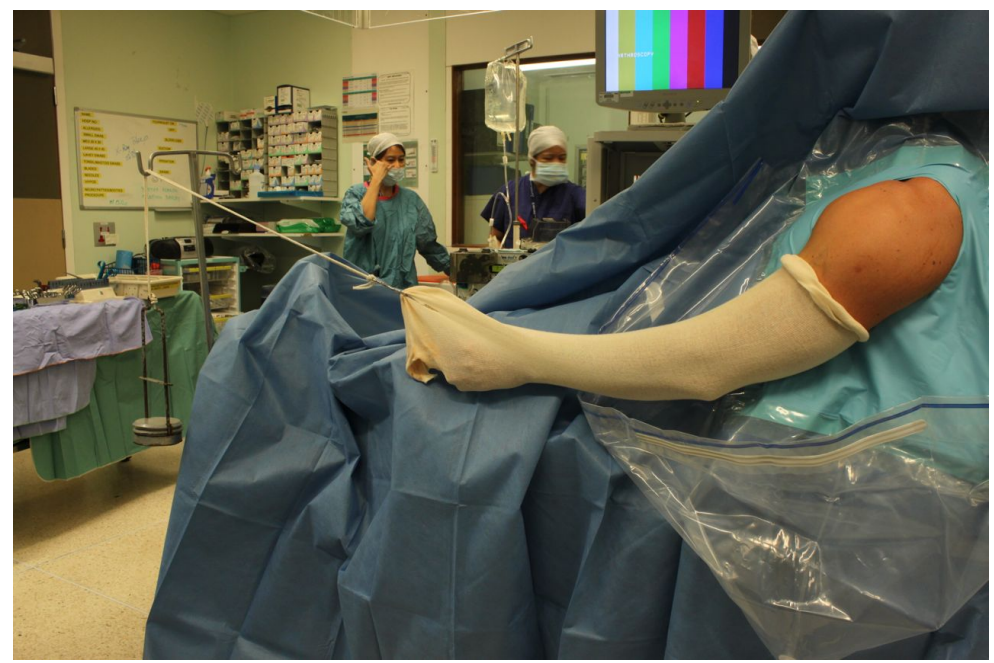

Figure 7. The set-up with drapes prior to final drape over unsterile area of hook through stockinette and its traction cord with weights.

\section{Discussion}

Though Friar's Balsam is discussed in the British National Formulary as an aromatic inhalant, it has been used successfully as a skin adhesive agent to permit the application of adhesive paper strips to geriatric skin where sutures are felt not to hold, specifically pre-tibial lacerations.

Friar's Balsam is also recommended for use as an antiseptic agent, making its adhesive properties all the more appealing during surgical procedures [5].

Though no specific mention of sterility is made regarding this product, it is by nature an antiseptic agent with a high alcohol content (96\%). The shelf life of Friar's Balsam is 36 months, and it can be stored at room temperature [6].

There have been reports of allergy [7] though in 10 years' use, we have yet to see contact dermatitis or anaphylaxis secondary to Friar's Balsam.

Other traction techniques involve large initial outlays and are described below:

The Notac Traction Kit [3] for an adult is $£ 5.38$ and for children is $£ 7.17$ without Value Added Tax (VAT). Costs for other consumables for traction using a boom arm attached to a clamp may also be incurred.

Padded traction requires several minutes of set-up time compared to several seconds with our technique, 
creating a further reduction in overall surgical time, which may itself enable more efficient use of theatre time.

One paper in the literature quoted a set-up time of 30 and 26 minutes with and without skin traction respectively, but we found our set-up times were significantly shorter, taking less than a minute per case to add traction.

The initial outlay for hardware varies depending on the type of traction selected by surgeon and/or team.

Shoulder suspension kits attached to boom arms and clamps are available for $£ 1000.00$ with "weightless" systems available for around $£ 3000$ [1].

The hydraulic SPIDER2 system from Smith \& Nephew [4] is available for approximately £13,000. The exact value for the total cost of consumables would be available on an individual institutional basis. This system is incredibly versatile and ideal for the unassisted surgeon where traction is essential for viewing and progress of the operation (stabilization and arthroplasty). Once the pedal is depressed, the system is free to move in any plane and this 3-dimensional versatility is highly appealing. On release of the pedal, the position of the arm is maintained, whether that is neutral or with traction applied.

Our system does permit good views with minimal cost both for initial hardware and ongoing consumable items, and to date has yet to fail when applied in the recommended fashion. Though not designed for this purpose, it is clear that in austere times, when hospitals are looking to cut costs, a department employing 2 shoulder surgeons who perform predominantly arthroscopic work averaging 350 cases per annum jointly may look to save several thousands of pounds compared to the next cheapest option using padded traction with a figure-of-8 crepe bandage attached to the drip stand, string and hook. Other systems' costs far exceed even designed-forpurpose skin traction.

Safe and effective application of the crepe bandage in a figure-of-8 fashion over padded traction equipment was deemed the surgeon's responsibility, since the theatre staffs were unable to reliably and reproducibly apply this. With our technique, we ensured that the surgeon or the theatre staffs preparing the skin were equally able, once taught, to apply the Friar's Balsam with stockinette and crepe bandage so that it never failed. This in itself is testament to the ease of use and reliability of this technique.

Institutions as large as each NHS trust can achieve significant discounts on products such as the consumables as well as the initial hardware required for the above traction technique. This suggests that any prices quoted are the maximum one would be expected to pay for any of the options discussed.

Posology would suggest that 10 - $15 \mathrm{ml}$ of Friar's Balsam is sufficient to achieve a thin layer on both dorsal and ventral surfaces of the arm, along the neck and on the chest. This would permit 3 aliquots per $50 \mathrm{ml}$ bottle, the size most widely available on the market.

\section{Conclusion}

Our system of traction for shoulder arthroscopy in the beach-chair position is cost effective, highly reproducible and easy to learn. We acknowledge that other systems have their advantages and disadvantages as discussed in the paper but would suggest that dissemination of this technique would be in the interest of surgeons across the globe.

\section{References}

[1] 3-Point Shoulder Distraction System. Cited 16 February 2014. http://www.arthrex.com/shoulder/3point-shoulder-distraction-system/products

[2] Peruto, C.M., Ciccotti, M.G. and Cohen, S.B. (2009) Shoulder Arthroscopy Positioning: Lateral Decubitus versus Beach Chair. Arthroscopy, 25, 891-896. http://dx.doi.org/10.1016/j.arthro.2008.10.003

[3] Mölnlycke Health Care—-Notac ${ }^{\text {TM }}$ Skin Traction Kit. Cited 16 February 2014. http://www.molnlycke.com/gb/Wound-Care-Products/Product-selector---Wound-division/Tabs/Products/Notac-Skin-T raction-Kit/?activeTab=2

[4] SPIDER2 Limb Positioner for Shoulder, Hip, Knee, and Extremities Surgery. Smith \& Nephew-US Professional, Cited 16 February 2014.

http://www.smith-nephew.com/professional/products/all-products/spider2-limb-positioner-for-shoulder--hip--knee--/

[5] Falkner-Heylings, J. (2012) Compound Tincture of Benzoin. Alliance Professional Development Programme, Cited 27 February 2014.

http://www.thealliancepsp.com/CPD/COMPOUND TINCTURE OF BENZOIN.pdf

[6] Friars’ Balsam BP-Summary of Product Characteristics (SPC)—(eMC) (2014) Datapharm Communication Ltd., 
Cited 27 February 2014. http://medicines.org.uk/emc/medicine/25211

[7] Scardamaglia, L., Nixon, R. and Fewings, J. (2003) Compound Tincture of Benzoin: A Common Contact Allergen? Australasian Journal of Dermatology, 44, 180-184. http://dx.doi.org/10.1046/j.1440-0960.2003.00674.X 